Fetal Diagnosis

and Therapy
Fetal Diagn Ther 2010;28:196-200

DOI: $\underline{10.1159 / 000320966}$
Received: March 17, 2010

Accepted after revision: September 2, 2010

Published online: October 20, 2010

\title{
Does Intervention in utero Preserve the Obstructed Kidneys of Fetal Lambs? A Radiological and Pathological Study
}

\author{
Junjie Sun ${ }^{\mathrm{a}}$ Fenghua Wang ${ }^{\mathrm{b}}$ Gaoyan Deng ${ }^{\mathrm{a}}$ Jiacong $\mathrm{Mo}^{\mathrm{a}}$ \\ ${ }^{a}$ Department of Pediatric Surgery, First Affiliated Hospital, University of Sun Yat-Sen, and bepartment of Surgery, \\ Guangzhou Women and Children's Medical Centre, Guangzhou, China
}

\section{Key Words}

Ureteropelvic junction obstruction - Fetal hydronephrosis • Radiological and pathological study $\cdot$ Animal model

\begin{abstract}
Objective: Ureteropelvic junction obstruction is a common cause of end-stage pediatric nephropathy. Our aim was to investigate whether in utero decompression can influence its development. Methods: A silastic tube was tied around the superior segment of the left ureter to cause partial unilateral obstruction in 22 fetal lambs at $75-85$ days of gestation. Three weeks later, tubes were removed in 10 of the fetuses. A single sham procedure was performed on 4 control fetuses. Intravenous pyelography (IVP) and pathological evaluations were conducted in obstructed, decompressed and control subjects. Results: IVP revealed damaged renal function in obstructed subjects. Macroscopically, obstructed kidneys were larger but weighed less and had thinned parenchyma. Microscopy revealed cortical cysts of various sizes and interstitial fibrosis. The number of glomeruli was markedly decreased. In contrast, decompressed kidneys were visualized during IVP, and pathological changes were greatly ameliorated. Conclusions: Relief of obstruction in utero seems to prevent or attenuate development of nephropathy in lambs. Clinical application of this procedure should proceed with caution until further data are obtained.
\end{abstract}

Copyright $\odot 2010$ S. Karger AG, Basel

\section{Introduction}

Ureteropelvic junction obstruction (UPJO) is a common cause of fetal hydronephrosis, having an incidence of approximately 1 in 2,000 [1]. While renal function in most mild UPJO patients can remain stable for many years, severe deterioration requiring surgical treatment occurs in some cases. However, Murer et al. [2] found that recovery from renal injury following relief of UPJO is not assured in some children; the hypothesis is that in utero intervention may help these patients. Glick et al. [3] caused complete, temporary unilateral ureteral obstruction in fetal lambs, and then evaluated renal function and histopathology after delivery. They found that in utero decompression could reverse functional and prevent or greatly ameliorate development of renal dysplasia. Chevalier et al. [4] temporarily occluded one ureter of 1-dayold rats, and 28 days later observed markedly reduced tubular atrophy and interstitial fibrosis compared to controls that had remained obstructed. In both studies, relief of obstruction in utero preserved functional renal mass to a great extent. However, both involved complete unilateral ureteral occlusion, while most clinical cases are caused by partial obstruction.

This study aims to investigate whether relief of severe partial unilateral ureteral obstruction in utero attenuates development of nephropathy in lambs.

\section{KARGER}

Fax +4161306 1234 E-Mail karger@karger.ch www.karger.com

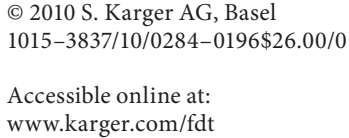

Dr. Mo Jiacong, $\mathrm{PhD}$

Department of Pediatric Surgery, First Affiliated Hospital

University of Sun Yat-Sen, Guangzhou 510080 (China)

Tel. +86203333 9931, Fax +862028823350

E-Mail gdmjc@ hotmail.com 
Table 1. Size, cortical thickness, weight, and number of glomeruli of kidneys in different groups (mean $\pm \mathrm{SD}$ )

\begin{tabular}{lrrrr}
\hline & \multicolumn{1}{c}{ CK } & OK & \multicolumn{1}{c}{ DK } & \multicolumn{1}{c}{$\mathrm{p}$} \\
\hline Size, $\mathrm{cm}^{2}$ & $8.54 \pm 0.85$ & $27.81 \pm 2.30$ & $8.12 \pm 0.80$ & $<0.05$ \\
Cortical thickness, cm & $0.64 \pm 0.05$ & $0.17 \pm 0.05$ & $0.60 \pm 0.08$ & $<0.05$ \\
Weight, g & $12.61 \pm 0.67$ & $5.91 \pm 0.70$ & $12.44 \pm 0.68$ & $<0.05$ \\
Glomeruli, n & $6.38 \pm 1.06$ & $1.89 \pm 0.60$ & $5.75 \pm 1.04$ & $<0.05$ \\
\hline
\end{tabular}

$\mathrm{CK}$ = Control kidneys; OK = obstructed kidneys; DK = decompressed kidneys.

\section{Materials and Methods}

\section{Animal Model}

Severe unilateral partial ureteral obstruction was induced in 22 fetal lambs at 75-85 days of gestation (full-term is 150 days) as follows: pregnant ewes were anesthetized with intravenous ketamine $3 \mathrm{mg} / \mathrm{kg}$ and $3 \%$ pentobarbital $0.4 \mathrm{ml} / \mathrm{kg}$. Open hysterotomy was performed via a flank laparotomy using a transperitoneal approach and the fetal lamb hindquarters were exteriorized. The superior portion of the left ureter was exposed and then partially obstructed by wrapping with an F6 split silastic tube. Methylthioninium chloride $(2 \mathrm{ml})$ was injected into the renal pelvis and its flow was observed to ensure that the obstruction was partial. The fetal abdomen was closed with a continuous monolayer suture and the fetus was repositioned. The uterine cavity was then closed with a continuous bilayer suture and the maternal abdominal wall with a layer-by-layer suture.

After the procedure, the ewes were taken to the ward and given free access to water and grass along with injections of maxipime ( $1 \mathrm{~g} /$ day) for infection prophylaxis and intramuscular injections of progesterone $(20 \mathrm{mg} /$ day) for miscarriage prevention.

Three weeks later, 10 of the 22 fetuses underwent another procedure following previously described surgical protocol for decompression, in which the silastic tube obstruction was removed. In addition, 4 fetal lambs underwent one sham procedure at 75-85 days of gestation and were used as controls. Lambs viable following full-term births were included in the study: aborted fetuses were excluded.

All protocols were approved by the Medical Ethics Committee of the First Hospital, which is affiliated with Sun Yat-Sen University.

\section{Functional Radiological Studies}

Intravenous pyelography (IVP) studies were performed on 5to 7-day-old study animals after administration of intramuscular anesthesia (846 composition $0.05 \mathrm{ml} / \mathrm{kg}$ ). Contrast dye (38\% urografin, $1.5 \mathrm{ml} / \mathrm{kg}$ ) was injected into an inguinal vein, an abdominal bandage was applied and the lamb was secured in the supine position. A series of abdominal X-rays were obtained, one every 5 $\min$ for 10 .

\section{Gross Pathology}

The lambs were sacrificed at the end of the IVP study. Kidneys were measured and then hemisected in the coronal plane. Sizes were recorded as length multiplied by width. Weights and the cortical thickness were measured and recorded.

\section{Histological Studies}

Hemisected organs were fixed in a $10 \%$ formaldehyde solution for $24 \mathrm{~h}$ at room temperature, and then embedded in paraffin, sliced along the coronal plane into $5-\mu \mathrm{m}$ sections and stained with hematoxylin and eosin. Sections were then observed under an Olympus CX4 light microscope. The number of glomeruli was determined by the radial glomerular count method [5].

Statistical Analysis

Results were expressed as mean \pm SD. SPSS 13.0 software (SPSS Inc., Chicago, Ill., USA) was utilized for one-way ANOVA analysis of the data. Significance was defined as $p<0.05$.

\section{Results}

The survival rate in this study was $80.8 \%$ (21/26): 9 in the obstructed group, 8 in the decompressed group, and all 4 in the control group.

During decompression procedures, obvious pyeloureterectasis was observed (ureteral diameter $>7 \mathrm{~mm}$ above and $<2 \mathrm{~mm}$ below the obstruction). Following silastic tube removal, inferior ureteral segments filled immediately. The kidneys of animals that were not decompressed appeared distended on gross examination at the end of the study.

\section{Functional Radiological Studies}

IVP was performed satisfactorily in 18 lambs but was not successful in 3 animals - 2 decompressed and 1 obstructed. In the 4 control and 6 decompressed lambs, both kidneys were visualized 10-20 min after contrast dye injection; but in the other 8 animals, while contralateral kidneys were visualized within $20 \mathrm{~min}$, the obstructed kidneys had not filled $45 \mathrm{~min}$ later (fig. 1). In all, 0/9 obstructed kidneys, 6/8 decompressed kidneys and 4/4 control kidneys were visualized within $20 \mathrm{~min}$ after contrast dye injection, which showed significant statistical difference.

\section{Gross Pathology}

Gross pathological data are presented in table 1 and figure 2 . The obstructed kidneys were significantly en- 
Fig. 1. The contralateral kidney was visualized clearly, while the obstructed kidney could not be seen even $45 \mathrm{~min}$ after the injection of contrast dye (a). Both the decompressed kidney and the contralateral kidney were visualized distinctly $20 \mathrm{~min}$ after the injection of contrast dye (b). R = Right; $\mathrm{L}=$ left.

Fig. 2. Morphological changes in gross samples (coronal section). Compared with the control kidneys (a), the obstructed kidney continued to enlarge and the medulla as well as the cortex became much thinner and the whole kidney became a sac (b). Relief of the obstruction in utero nearly reversed the kidney size and retained the cortical thickness (c). CK = Control kidneys; OK = obstructed kidneys; DK = decompressed kidneys; $\mathrm{KCO}=$ kidneys contralateral to the obstructed ones; $\mathrm{KCD}=$ kidneys contralateral to the decompressed ones.
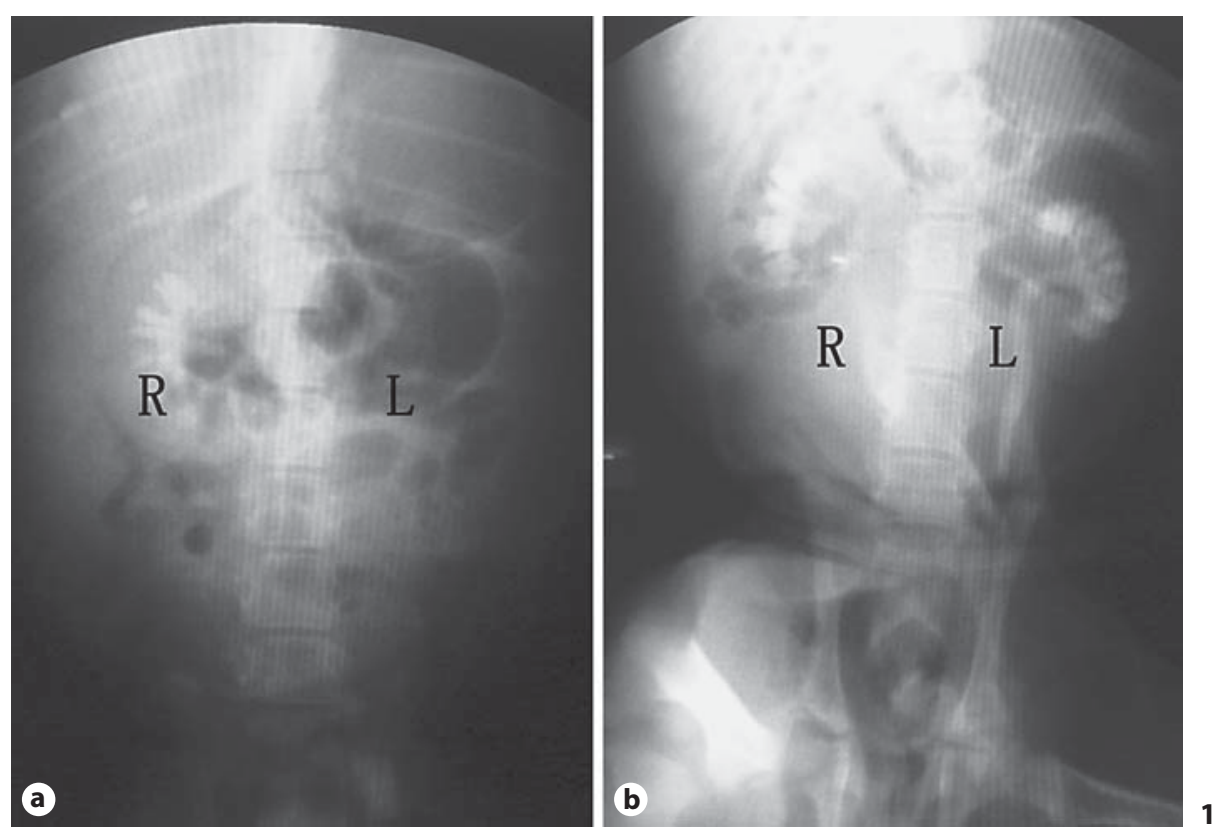

larged but were lighter in weight and had thinner cortices compared with control kidneys $(\mathrm{p}<0.05)$. Relief of obstruction reversed changes in these parameters compared with obstructed kidneys ( $\mathrm{p}<0.05)$, and no differences were seen between or among the control kidneys and contralateral organs from the obstructed and decompressed groups $(\mathrm{p}>0.05)$. To be addressed, the three described parameters, size, cortical thickness, and weights were normally distributed.

\section{Histological Studies}

Microscopically no dysplastic changes were observed and basic elements appeared intact in all cases. However, in the obstructed specimens, cysts of various sizes had formed in the renal parenchyma and interstitial tissue fibrosis was observed. Furthermore, some glomerular capillary loops had collapsed and the number of glomeruli was much decreased compared with control kidneys $(\mathrm{p}<0.05)$.

Decompression reversed some of the histological changes. Glomerular capillary loop collapse and dilatation of renal tubules and Bowman's capsule were greatly ameliorated. Importantly, relieving obstruction resulted in preservation of the number of glomeruli (decompressed vs. obstructed, $\mathrm{p}<0.05)$. However, the decompressed kidneys contained deposits of extracellular matrix (fig. 3).
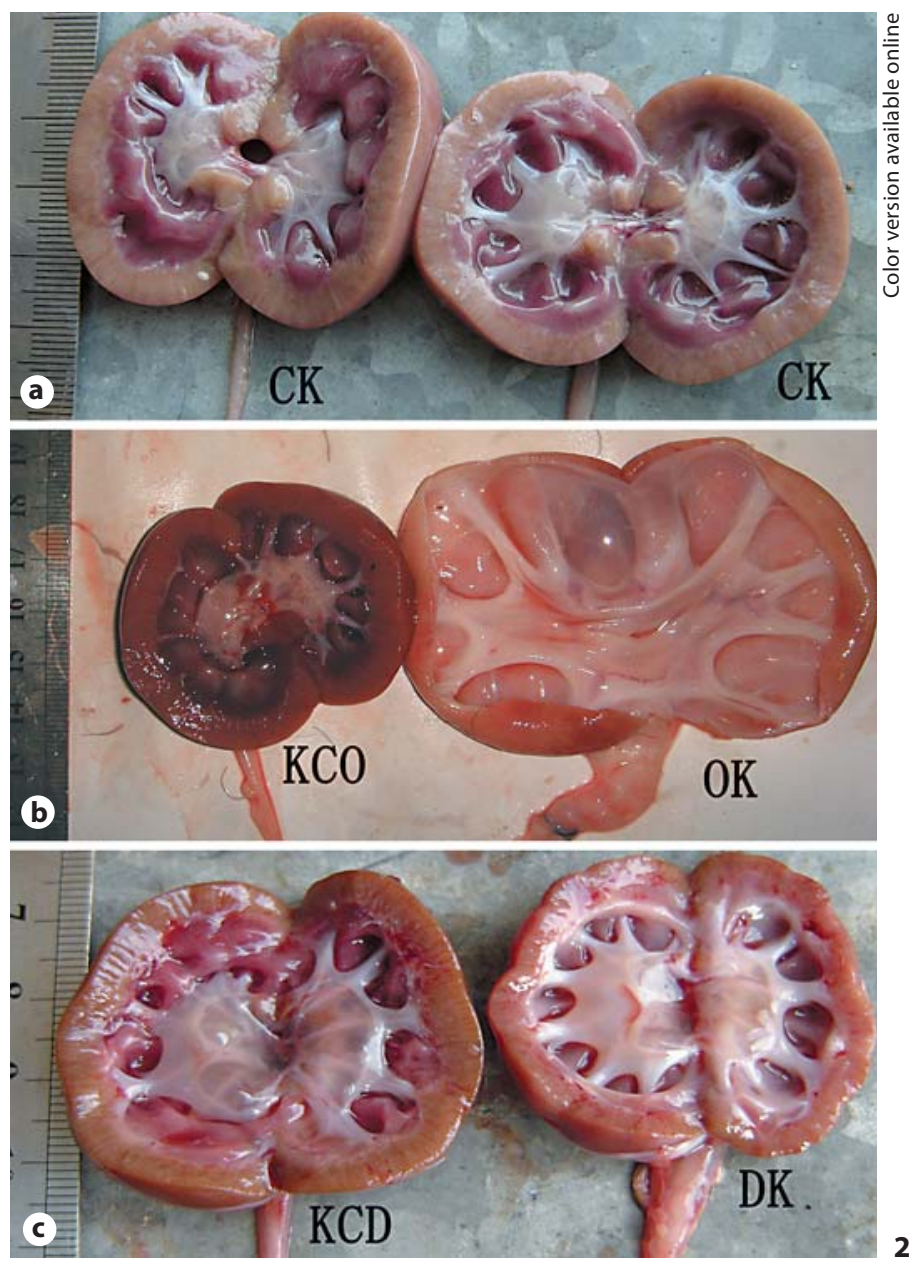

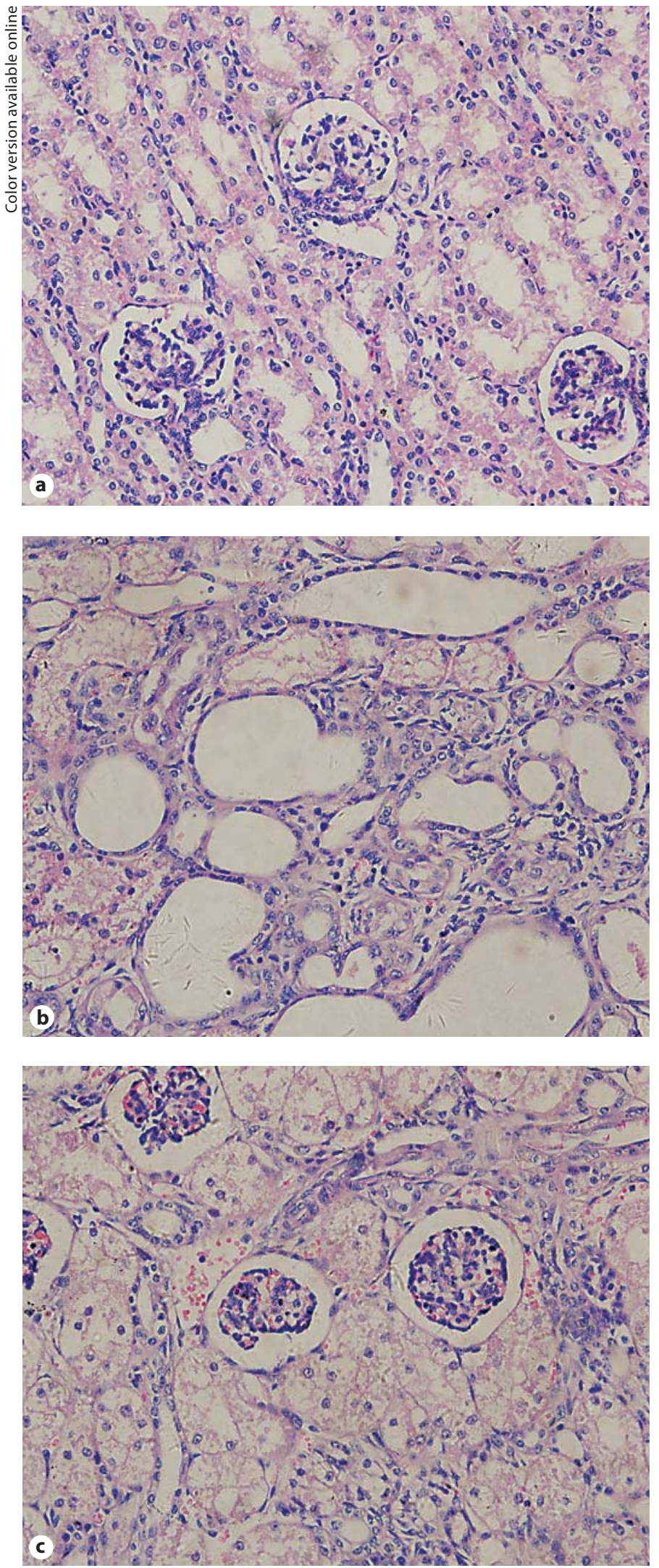

\section{Discussion}

Since Harrison et al. [6] in 1981 reported the first successful in utero decompression for hydronephrosis, such interventions have been attempted with increasing frequency. To date, fetal surgery has been applied clinically in a selected group of disorders including, among others, bilateral urinary tract obstruction (UTO), diaphragmatic hernia, and congenital cystic adenomatoid malformation. Nearly all these disorders are life-threatening, and performing fetal surgery can ameliorate suffering after birth. With continuing development of anesthetic, surgical, and tocolytic techniques, not only are life-threatening disorders potential candidates for fetal surgery, but increasingly lesions that are irreversible postnatally will become indications for in utero intervention. According to Stephen [7], delaying surgery to correct UPJO may keep ipsilateral renal function from returning to normal, or even from improving significantly, and congenital hydronephrosis fails to improve postnatally in as many as $40 \%$ of cases. Consequently, fetal surgery should be considered for severe UPJO. Unfortunately, very few studies have been conducted to date, so the effectiveness of this intervention is unknown. Results from the present study should provide impetus for funding future investigations.

The type of renal pathological change depends on the completeness of obstruction and its timing with respect to the stage of glomerulogenesis. Kitagawa [8] ligated the urethra and urachus of fetal lambs at 60 and 90 days of gestation and found definite dysplastic changes in the former group, but none in the latter. Wen et al. [9] created mild or severe partial UTO in 2-day-old rats. Several months later, renal dysplasia was observed in the severe partial UTO group, while none was seen in the mild partial UTO group. In this study, we induced severe partial UTO in fetal lambs at 75-85 days of gestation, and all obstructed kidneys developed hydronephrosis or cystic changes, but no dysplasia was observed.

When UTO is relieved in utero, pathological changes can be ameliorated. Especially striking is the finding that

Fig. 3. Histological changes observed in various kidneys. Compared with the control kidneys (a), cysts of various sizes, blood vessel loops collapse, fibrosis, and decreased number of glomeruli were observed in the obstructed kidneys (b). The number of glomeruli was retained and the saccular ectasia was greatly ameliorated in the decompressed kidneys $(\mathrm{C}) . \times 400$. 
cortical thickness is preserved and the number of glomeruli remains stable, observations in accordance with previous reports. Edouga et al. [10] created bladder outlet obstruction in fetal lambs at 60 days of gestation, decompressed at 90 days, and then determined outcomes for obstructed, shunted, and control fetuses at 120 days of gestation. The number of glomeruli decreased in obstructed, but not shunted, fetuses. In the current study, other histological changes, such as blood vessel loop collapse, renal tubule dilatation, and Bowman's capsule dilatation, were greatly ameliorated by decompression, but extracellular matrix deposits were present in these kidneys. In addition, we observed a trend toward increased size and weight in the kidneys contralateral to the obstructed kidneys, as compared with controls, but the trend did not reach statistical significance in this study. If validated in the future, this finding might be explained by the phenomenon of compensatory renal hypertrophy, which occurs in the setting of contralateral nephropathy. Further studies are needed to elucidate possible mechanisms underlying all of these phenomena.

IVP is a common clinical radiological technique that is indicative of renal function. IV contrast dye is filtered by functioning glomeruli, and then the urinary tract can be visualized on X-ray. IVP is readily performed on anesthetized animals and is a convincing evaluation of renal function. In the present study, no urinary tract visualization was found in fetuses with obstructed kidneys, so re- nal function had been adversely affected. As mentioned above, Edouga et al. [10] created partial bladder obstruction in fetal lambs at 60 days of gestation and found decreased renal function 60 days later. In contrast, Bogaerta et al. [11] created partial bladder obstruction in fetal lambs at 90 days of gestation, determined glomerular filtration rate and urine volume 30-35 days later, and found no evidence of nephropathy. It appears that earlier obstruction causes more damage, and that the longer obstruction continues the more severe is the decrease in renal function. In any case, in these studies, antenatal relief of obstruction seems to have been key to accomplishing favorable postnatal outcomes.

\section{Conclusions}

Our study supports the hypothesis that relief of UTO in utero can alleviate development of nephropathy. However, extrapolation of these findings to clinical practice should be done with caution until further evidence is available.

\section{Acknowledgement}

The study is supported by the Hall of Science and Technology, Guangdong Province, China.

\section{References}

1 Becker A, Baum M: Obstructive uropathy. Early Hum Dev 2006;82:15-20.

$\checkmark 2$ Murer L, Benetti E, Centi S, Artifoni L, Capizzi A, Zucchetta P: Clinical and molecular markers of chronic interstitial nephropathy in congenital unilateral ureteropelvic junction obstruction. J Urol 2006;76:26682673.

-3 Glick PL, Harrison MR, Adzick NS, Noall RA, Villa RL: Correction of congenital hydronephrosis in utero. IV. In utero decompression prevents renal dysplasia. J Pediatr Surg 1984;19:649-656.

4 Chevalier RL, Thornhill BA, Wolstenholme JT, Kim A: Unilateral ureteral obstruction in early development alters renal growth: dependence on the duration of obstruction. J Urol 1999;161:309-313.
Rodriguez MM: Development renal pathology: its past, present, and future. Fetal Pediatr Pathol 2004;23:211-229.

6 Harrison MR, Golbus MS, Filly RA, Callen PW, Katz M, de Lorimier AA: Fetal surgery for congenital hydronephrosis. N Engl J Med 1982;306:591-593.

7 Stephen AK: Neonatal management of unilateral hydronephrosis: role for delayed intervention. Urol Clin North Am 1998;25: $181-186$
8 Kitagawa $\mathrm{H}$ : The pathogenesis of dysplastic kidney in a urinary tract obstruction in the female fetal lamb. J Pediatr Surg 1999;34: 1678-1683.

9 Wen JG, Frokier J, Zhao JB, Ringgaard S, Jorgensen TM, Djurhuus JC: Severe partial ureteric obstruction in newborn rats can produce renal dysplasia. BJU Int 2002;89: 740-745.

10 Edougav D, Hugueny B, Gasser B, Bussieres L, Laborde K: Recovery after relief of fetal urinary obstruction: morphological, functional and molecular aspects. Am J Physiol Renal Physiol 2001;281:26-37.

- 11 Bogaerta GA, Gluckmana GR, Mevorach RA, Kogan BA: Renal preservation despite 35 days of partial bladder obstruction in the fetal lamb. J Urol 1995;154:694-699. 